\title{
Supporting Japanese Mothers of Children at Risk for Attention Deficit Hyperactivity Disorder (ADHD): A Small Scale Randomized Control Trial of Well Parent Japan
}

\author{
Shizuka Shimabukuro ${ }^{1}$ David Daley ${ }^{2,3} \cdot$ Margaret Thompson $^{4,5} \cdot$ Cathy Laver-Bradbury $^{4} \cdot$ Kaoruko Lovern $^{1}$. \\ Gail Tripp ${ }^{1}$
}

Published online: 3 March 2020

(c) The Author(s) 2020

\begin{abstract}
Objectives Guidelines recommend parent management training in the multi-modal treatment of attention deficit hyperactivity disorder (ADHD). The availability of such interventions in Japan is limited. This study evaluated the effects of Well Parent Japan, a hybrid intervention including a group Japanese language adaptation of the New Forest Parenting Programme for ADHD (NFPP) augmented with strategies to improve parent's psychological wellbeing and enhance confidence in their ability to implement change.

Methods Mothers of children aged 6-12 years displaying marked symptoms of ADHD were randomly assigned to the intervention $(n=28)$ or a waitlist control condition $(n=24)$. Measures were completed at baseline and again 14 weeks later. Parenting stress was the primary outcome. Other outcomes included maternal depression, parenting self-efficacy, reported and observed parenting behaviour, and maternal ratings of child ADHD, oppositional defiant disorder (ODD) symptoms, and internalizing problems.

Results Analysis of covariance (ANCOVA) compared the groups post intervention. Intervention mothers reported significantly less parenting stress, higher parenting self-esteem and use of more effective parenting strategies compared with controls, including a reduction in observed negative parenting, post intervention. These mothers also reported lower levels of child aggression and internalizing problems post intervention together with a trend $(p<0.05)$ toward reduced symptoms of inattention.

Conclusions Well Parent Japan is an effective psychosocial intervention for parents of children with ADHD in Japan. The group format and the session content is well tolerated. This is the first randomized control trial (RCT) of a psychosocial intervention targeting ADHD in Japan.
\end{abstract}

Keywords ADHD $\cdot$ Parenting $\cdot$ NFPP $\cdot$ Wellbeing $\cdot$ Japan

\section{Highlights}

- Well Parent Japan reduced mothers' parenting stress and increased their efficacy.

- Program completion also resulted in improvements in observed and reported parenting practices.

- Mothers reported reduced ADHD symptoms, aggression, and internalizing problems.

- Well Parent Japan offers an effective psychosocial intervention for ADHD in Japan.

Gail Tripp

tripp@ oist.jp

1 Human Developmental Neurobiology Research Unit, Okinawa Institute of Science and Technology Graduate University, 1919-1 Tancha, Onna-son, Okinawa 904-0495, Japan

2 Division of Psychiatry and Applied Psychology, School of Medicine, University of Nottingham, Nottingham NG7 2TU, UK

3 NIHR MindTech Healthcare Technology Cooperative \& Centre for ADHD and Neurodevelopmental Disorders Across the Lifespan CANDAL, Institute of Mental Health, University of Nottingham Innovation Park, Triumph Road, Nottingham NG7 2TU, UK

4 Academic Unit of Psychology, University of Southampton, Building 44 Highfield Campus, Southampton SO17 1BJ, UK

5 CAMHS, Solent NHS Trust, Better Care Centre, Western Community Hospital, William MacLeod Way, Southampton SO16 4XE, UK 
Attention Deficit Hyperactivity Disorder (ADHD) is a common neurodevelopmental disorder with an estimated worldwide prevalence of approximately 5\% (American Psychiatric Association 2013). Prevalence estimates in Japan range from $2.5 \%$ (Japanese Ministry of Education, Culture, Sports, Science and Technology 2012) to $10 \%$ (Sakakibara 2007). The disorder is associated with numerous functional impairments and poor long-term outcomes including reduced academic achievement, employment difficulties and strained social relationships (Daley and Birchwood 2010; Mikami et al. 2007). Having a child with ADHD also negatively impacts parents' emotional wellbeing, parenting practices and the parent-child relationship (Theule et al. 2013). Effective interventions for children with ADHD and their families are a high priority (Shaw et al. 2012). The limited research available regarding the impact of ADHD on Japanese families would suggest they experience similar levels of symptoms, problems and impairments (Shimabukuro et al. 2017).

Parenting interventions are recommended for ADHD (National Institute for Health and Care Excellence 2018), with proven efficacy in numerous randomized control trials (RCT, Daley et al. 2014; Sonuga-Barke et al. 2013). These interventions typically provide parents with behavioural strategies aimed at increasing the frequency of desired behaviour, whilst reducing the frequency of unwanted behaviour. Probably blinded, i.e., raters unaware of the intervention, outcome measures indicate these interventions benefit parenting practices and improve conduct problems in children with ADHD as well as parent reported ADHD symptoms. Ratings of children's emotional, social and academic functioning and parent self-worth also show change, although most studies have not included blinded outcomes for these variables (Daley et al. 2018). A small number of studies have directly targeted parental wellbeing, specifically maternal depression (Chronis et al. 2006; Chronis et al. 2013) and parenting stress (Treacy et al. 2005) in parents of children with ADHD. These studies report significant improvements in parents' emotional functioning.

The availability of psychosocial interventions for ADHD in Japan is limited (Takayama 2008). Two parenttraining programmes have been widely implemented in Japan, the Hizen Parenting Skills Training developed in Japan for children with Autism and Intellectual Disability (HPST, Okuma and Ito 2005), and the Seiken parenting program (Iwasaka 2012) which was adapted from American interventions (Barkley 2013; Whitham 1998) which focused on more generic aspects of parenting (see Shimabukuro et al. 2017). Both programmes are used in the management of a wide range of disorders/disabilities, including ADHD. To date these programmes have not been subjected to rigorous evaluation through RCTs and lack a robust evidence base for treating ADHD symptoms and associated difficulties.

The eight-session group Positive Parenting Program (Triple P, Sanders et al. 2003) has been evaluated in RCTs with Japanese families of children with mild behaviour problems living in Australia (Matsumoto et al. 2007) and in Metropolitan Japan (Matsumoto et al. 2010). These studies report improvements in parent reported child behaviour, parenting behaviour and parenting competence. To date, this programme has not been evaluated in an RCT with Japanese families of children with ADHD. None-the-less, the findings from these two studies suggest Western style parenting interventions can be successfully adapted for use with Japanese families.

Feedback from Japanese mothers of children with ADHD, who participated in a pilot study of a standard behavioural intervention coupled with two parent support sessions, indicated that they approved the group format, while preferring it to be limited to mothers to promote honest discussion; perceived a need for additional psychoeducation about ADHD to help reduce self-blame and understand why their children behave in the ways they do; requested additional specific parent support sessions, and training methods to address their child's ADHD related difficulties, e.g., poor working memory; and believed role play was helpful in supporting their acquisition of key skills. Group leaders perceived a need for additional explanation of behavioural strategies especially those inconsistent with Japanese parenting practices, e.g., the importance of using praise, and a need to enhance mothers communication skills (Shimabukuro et al. 2017).

In response to this feedback we adapted and extended the New Forest Parenting Programme (NFPP) for use with Japanese mothers of children with ADHD (Shimabukuro et al. 2017). The NFPP was chosen as it was specifically developed for the management of ADHD including ADHD specific psychoeducation and skill training to address ADHD related deficits, addressing important concerns of Japanese mothers (Shimabukuro et al. 2017). Like other recommended interventions, it includes behavioural strategies aimed at helping parents manage oppositional and defiant behaviour together with games and activities targeting neuropsychological deficits often present in children with ADHD, e.g. working memory (Sonuga-Barke et al. 2006). A key concept underlying NFPP is that by engaging with their child in such activities, parents can help to support the development of their child's cognition and selfregulation (Thompson et al. 2009). Four RCTs have shown receipt of therapist-led NFPP is associated with a reduction in parent reported ADHD symptoms (Abikoff et al. 2015; Sonuga-Barke et al. 2018; Thompson et al. 2009) and improvements in parental well-being (Sonuga-Barke et al. 2001). It has also been shown to be effective in routine 
clinical care when compared against intensive treatment as usual (Lange et al. 2018).

In adapting NFPP for use with Japanese families, the eight-session group-based programme (Laver-Bradbury and Harris 2009) was combined with additional sessions to increase mothers understanding of ADHD, address their psychological well-being (Treacy et al. 2005) and enhance confidence in their ability to implement change. Despite increased public recognition of the challenges of parenting children with neurodevelopmental disorders, cultural expectations in Japan do not provide strong support for mothers sharing parenting or personal difficulties outside of the family. The traditional Japanese parenting context, which emphasizes effort and self-reflection on the part of the child, and where criticism is used to encourage selfmonitoring/self-improvement, often leads to some family resistance to the introduction of "Western" style parenting approaches. Traditionally mothers bear the majority of responsibility for their child's difficulties. These cultural and social factors make strengthening mothers' emotional wellbeing, and enhancing their confidence to effect change, especially important for the effective implementation of the skills taught in NFPP in Japan. The process of adapting NFPP is reported in Shimabukuro et al. (2017). All modifications to, and translation of, NFPP materials were undertaken in consultation with the NFPP developers.

These additional sessions, presented prior to the NFPP training, included: further psycho-education about ADHD, stress management training, training in cognitive restructuring, effective communication, and problem-solving. Treacy et al. (2005) and Chronis-Tuscano et al. (2013) demonstrate the value of such interventions in improving the psychological functioning of parents of children with ADHD. In delivering this hybrid programme the additional psychoeducation was designed to help mothers appreciate why traditional Japanese parenting practices might not be effective for children with ADHD. This included repeated discussions of the need to reduce criticism in favor of praise. Mothers were encouraged to discuss their discomfort/uncertainty with implementing Western parenting strategies. More didactic teaching practices and role plays were used to give mothers confidence to use new parenting strategies.

The results of the pre-post proof of concept study, based on data from 17 Japanese mothers who completed the intervention, are promising (Shimabukuro et al. 2017). Participating mothers reported a decrease in children's ADHD symptoms and aggression, more effective parenting practices and reduced parenting stress. These preliminary findings require confirmation in an RCT. The current study evaluates the efficacy of the hybrid intervention, referred to here as Well Parent Japan, with Japanese mothers of children diagnosed with ADHD or displaying marked symptoms of the disorder in an RCT. The study targets mothers only in response to the stated preferences of mothers who participated in the initial pilot study (Shimabukuro et al. 2017). If successful, Well Parent Japan will provide a culturally accessible parenting intervention for use with Japanese families of children with ADHD. We believe this is the first RCT of a parenting programme targeting ADHD in Japan. Importantly the programme was adapted for use with Japanese families through partnering with the original programme developers and Japanese consumers in the community. The result is a programme pairing strategies addressing mothers' psychological readiness to make change with proven parenting strategies for addressing ADHD symptoms. The number of behavioural parenting programmes including such skills training remains limited worldwide (see Barlow et al. 2014 for a review).

Based on the results of earlier randomized trials of NFPP, and the findings from the Japanese proof of concept study, participation in Well Parent Japan is predicted to reduce parenting stress and parent reported symptoms of ADHD, aggression and internalizing problems, and to improve parenting practices and parenting self-esteem. As per the trial registration (ISRCTN Register, ISRCTN71988966, http://isrctn.org) the primary outcome for the current study is parenting stress with other aspects of parent psychological functioning, parenting style and mothers' reports of the children's ADHD symptoms, ODD symptoms, aggression and internalizing problems secondary outcomes. Sample estimates for reductions in parenting stress were based on effect sizes reported by Treacy et al. (2005). Stress was chosen as the primary outcome as previous metaanalyses of behavioural interventions for ADHD (Daley et al. 2014) have indicated that behavioural interventions do not impact on parental wellbeing, and in this study the intervention was specifically designed to target both the mother's wellbeing and the child's ADHD behaviour.

\section{Method}

\section{Participants}

Mothers were recruited in four cohorts between September 2014 and April 2017 through a range of sources (including advertisements and public announcements). They completed and returned Japanese language versions of the Swanson, Nolan \& Pelham rating scale (SNAP, Swanson 1992) and the Gilliam Autism Rating Scale 3rd Edition (GARS-3, Gilliam 2013). They then participated in semistructured telephone interviews by the first author to confirm the nature of their child's difficulties, including review of their questionnaire ratings, and to assess their, i.e., the mothers, suitability for inclusion in a group parenting programme. When sufficient participants meeting the inclusion 
Table 1 Parent and child demographic and diagnostic characteristics, analyses, and significance

\begin{tabular}{|c|c|c|c|c|c|c|}
\hline \multirow{2}{*}{$\begin{array}{l}\text { Variable } \\
\text { Parent }\end{array}$} & \multicolumn{2}{|c|}{$\begin{array}{l}\text { Immediate } \\
\text { Treatment Group }\end{array}$} & \multicolumn{2}{|c|}{$\begin{array}{l}\text { Waitlist } \\
\text { Control Group }\end{array}$} & \multirow[t]{2}{*}{ Analysis } & \multirow[t]{2}{*}{$p$ value } \\
\hline & & & & & & \\
\hline Age (M, SD) & 40.59 & 4.06 & 42.10 & 4.46 & $t(46)=-1.219$ & $p=0.229$ \\
\hline \multicolumn{7}{|l|}{ Education $(\mathrm{n}, \%)$} \\
\hline High School & 9 & 33.33 & 5 & 23.81 & $x^{2}(2, N=47)=4.78$ & $p=0.092$ \\
\hline Junior College/Technical & 8 & 29.63 & 12 & 57.14 & & \\
\hline Degree/Postgraduate & 10 & 37.04 & 3 & 14.29 & & \\
\hline \multicolumn{7}{|l|}{ Family situation (n, \%) } \\
\hline Two-parent family & 21 & 77.78 & 17 & 80.95 & $x^{2}(1, N=48)=0.72$ & $p=0.788$ \\
\hline Single-parent family & 6 & 22.22 & 4 & 19.05 & & \\
\hline \multicolumn{7}{|l|}{ Child } \\
\hline Age $(\mathrm{M}, \mathrm{SD})$ & 8.04 & 1.61 & 8.86 & 1.68 & $t(46)=-1.720$ & $p=0.092$ \\
\hline Estimated IQ (M, SD) & 101.26 & 16.92 & 99.00 & 16.79 & $t(44)=0.447$ & $p=0.657$ \\
\hline Gender (n boys, $\%$ ) & 21 & 77.78 & 19 & 90.48 & $x^{2}(1, N=48)=1.37$ & $p=0.242$ \\
\hline Comorbid PDD ${ }^{\mathrm{a}}$ (n yes, \%) & 5 & 18.52 & 4 & 19.05 & $x^{2}(1, N=48)=1.57$ & $p=0.210$ \\
\hline Medication (n yes, \%) & 5 & 18.52 & 4 & 19.05 & $x^{2}(1, N=48)=0.002$ & $p=0.963$ \\
\hline Support Services (n yes, \%) & 19 & 70.37 & 9 & 42.86 & $x^{2}(1, N=47)=0.046$ & $p=0.831$ \\
\hline \multicolumn{7}{|l|}{ Education (n, \%) } \\
\hline Regular classroom & 21 & 77.78 & 17 & 80.95 & $x^{2}(1, N=48)=1.99$ & $p=0.575$ \\
\hline Special classroom & - & 18.52 & 1 & 4.76 & & \\
\hline Mixed & 5 & 3.70 & 2 & 9.52 & & \\
\hline Other & 1 & & 1 & 4.76 & & \\
\hline \multicolumn{7}{|l|}{ Time $1 \mathrm{SNAP}^{\mathrm{b}}(\mathrm{M}, \mathrm{SD})$} \\
\hline Inattention severity & 18.89 & 3.83 & 19.33 & 3.95 & $t(46)=-0.393$ & $p=0.696$ \\
\hline Hyperactivity/Impulsivity severity & 14.15 & 5.68 & 11.62 & 6.20 & $t(46)=1.469$ & $p=0.149$ \\
\hline ODD severity & 9.70 & 6.12 & 9.67 & 4.78 & $t(46)=0.023$ & $p=0.982$ \\
\hline
\end{tabular}

${ }^{\mathrm{a}}$ Reported by parent

${ }^{\mathrm{b}}$ Severity scores based of sum of item scores criteria were recruited they were randomized to immediate treatment or waitlist control groups by the first author using a simple random number generator.

Inclusion criteria were fluency in Japanese language and parenting a child, aged 6-12 years, demonstrating 6 or more definite symptoms of inattention and/or hyperactivity/ impulsivity on the parent completed SNAP. Due to service limitations, and the stigma attached to psychiatric diagnoses in Japan, not all parents seek formal diagnoses of ADHD. Exclusion criteria were: self-reported psychiatric symptomatology in the mother or other personal issues for which a group programme would be counter-indicated (e.g., delusions or paranoia, no parents were excluded); current or recent, i.e., within two months of screening, participation in another parenting programme; and the presence of moderate to severe Diagnostic and Statistical Manual of Mental Disorders (5th ed., DSM-5, APA 2013) symptoms of Autism Spectrum Disorder (ASD) in the target child, i.e., endorsement of symptoms equivalent to Level 3 on the GARS-3. Mild ASD and/or other reported comorbidities in the child did not exclude the mother from participating. For children taking medication for ADHD, mothers were asked to maintain the child's medication status throughout the trial. All families complied with this request. Demographic and available diagnostic information is presented in Table 1.

\section{Procedure}

Participants were asked to complete baseline measures prior to randomisation into the immediate treatment $(n=28)$ or waitlist control $(n=24)$ groups $(\mathrm{T} 1$; pre-intervention) and again 14 weeks later (T2; post-intervention for the treatment group) marking the end of the RCT. Mothers attended laboratory assessments with the target child at T1 and T2 during which they engaged in an interactive pasta making task, recorded for later coding. During the T1 assessment the child was also administered two subtests (Block Design and Vocabulary) from the Japanese Wechsler Intelligence Scale for Children-fourth edition (WISC-IV, Wechsler 2010). 
Fifty-two mothers met inclusion criteria and agreed to participate in the study. Twenty-eight mothers were randomly assigned to the immediate treatment group and 24 to the wait-list control group. All mothers assigned to the treatment group participated in the intervention. In the control group, three mothers withdrew, two after completing the pre-treatment questionnaires (one reported being too busy to participate, the other moved away from the area), another mother participated in the laboratory assessments but did not return the pre-treatment questionnaires and subsequently withdrew from the study. Figure 1 details participant flow through the study.

Immediate treatment group mothers participated in weekly Well Parent Japan sessions for 13-weeks. The waitlist control group had no further contact with the researchers until the T2 assessment 14-15 weeks later. They were then offered opportunity to participate in the Well Parent Japan intervention. Treatment and control parents were never included in the same treatment groups. Participating mothers received a 1000 Japanese yen voucher (US\$10.00) following each session to help cover transport costs.

Four treatment groups were run by the same two female therapists, native Japanese speakers with graduate degrees in psychology. The principal therapist completed the standard NFPP training programme in English in the UK. During the course of the RCT she participated in regular supervision with two of the NFPP developers. The second therapist was trained and supervised by the principal therapist.

Group sessions were $2 \mathrm{~h}$ in duration. If unable to attend a group session, participants were required to attend a catch-up session before the next group. A maximum of two catch-up sessions were offered per participant and no participants missed more than two group sessions. Fifteen mothers $(53.6 \%)$ attended all group sessions, eight mothers $(28.6 \%)$ missed one session and five mothers (17.9\%) missed two sessions. Immediate treatment groups ranged in size from five to eight mothers.

To maintain treatment fidelity, the two leaders ran the groups according to an approved translation of the English NFPP manual. As in other NFPP RCTs, the group leaders reviewed the topics to be covered prior to each session and reviewed session content and checked coverage following the session. Any material omitted from a session was presented at the beginning of the next group session. The primary therapist delivering the intervention received weekly supervision via Skype from the NFPP programme developers for the first three groups reducing to monthly supervision for the remainder of the trial. The content of Well Parent Japan is described in Appendix 1.

\section{Measures}

\section{Child behaviour}

ADHD and ODD symptoms The SNAP (Swanson 1992) is a 26-item rating scale assessing the presence and severity of symptoms of DSM-IV ADHD and ODD. If screening took place less than one month before the T1 assessment, the SNAP was administered at screening and T2 only. The measure was translated into Japanese for research in the Human

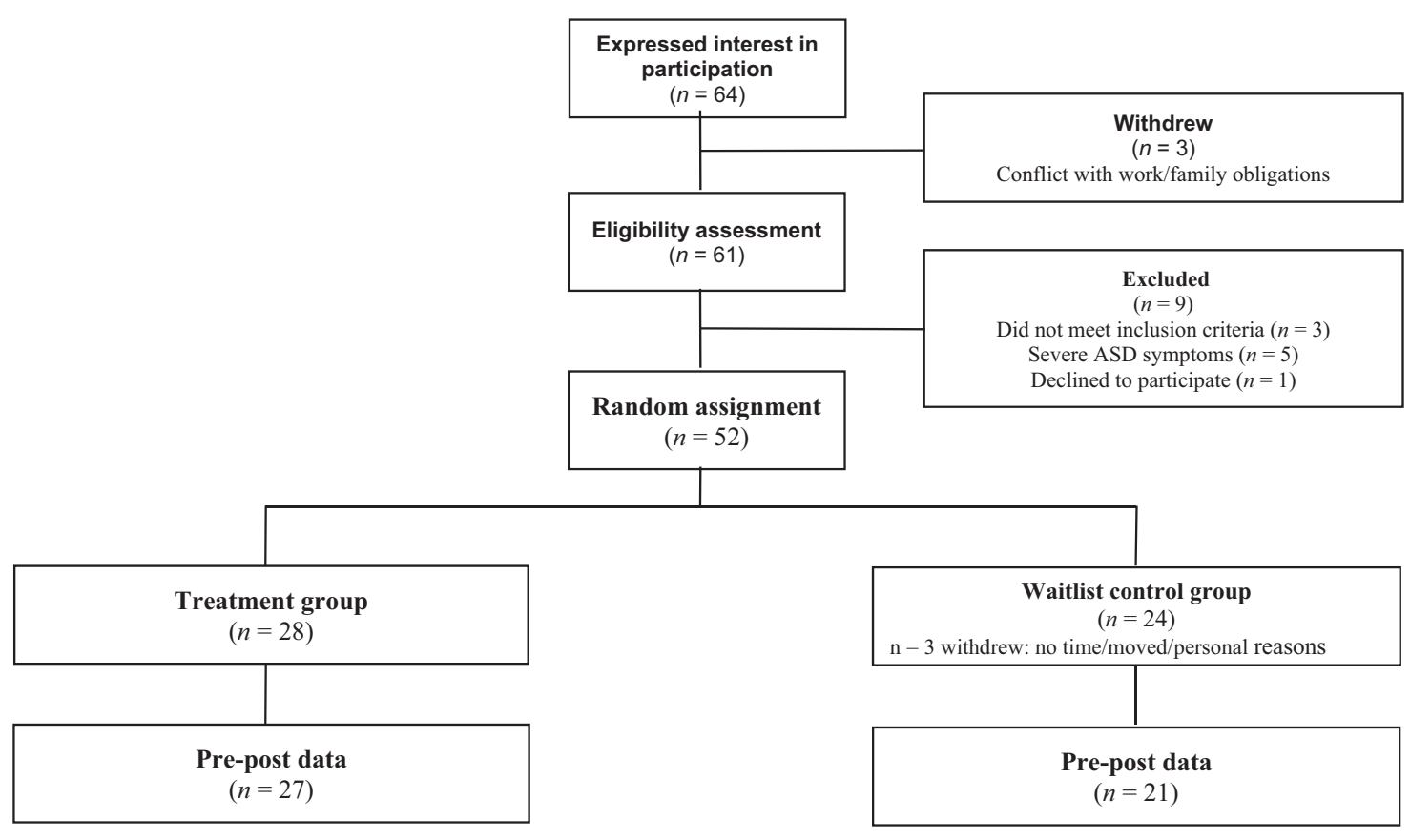

Fig. 1 Participant flow chart 
Developmental Neurobiology Unit with the permission of the scale's first author and in collaboration with the Japanese National Institute of Mental Health. The Japanese translation has excellent psychometric properties (Inoue et al. 2014). Cronbach's alpha in the current study was 0.80 for Inattention, 0.88 for Hyperactivity/Impulsivity, and 0.91 for ODD.

ASD symptoms The GARS-3 (Gilliam 2013) is a 58-item instrument designed to identify the presence and severity of symptoms of ASD. This measure was translated into Japanese for the current study with the permission of the developer. The translation and back translation were undertaken by the first author and a bilingual Japanese clinical psychologist licensed in the US. In the current study Cronbach's alpha ranged from 0.81 (Maladaptive Speech) to 0.90 (Social Interactions).

General behaviour problems/comorbidity The Child Behavior Checklist 4-18 (CBCL, Achenbach 1991) is a 113-item Broadband behaviour rating scale that assesses parent/caregiver perceptions of emotional and behavioural problems in children and adolescents. The measure includes eight problem scales and two broadband factors (Internalizing and Externalizing Behavior) and a Total Problems score. Three items $(2,4$, and 5$)$ differ between the original measure and the Japanese translation. The Japanese version of the CBCL/4-18 reports good psychometric properties (Itani et al. 2001). In the current study Cronbach's alpha for the Inattention and Aggression Problem scales was 0.61 and 0.85 respectively, and for Internalizing Behavior (sum of the Withdrawal, Somatic and Anxiety/Depression problem scales) 0.79 .

\section{Parent self-report}

Parenting stress The 78-item Japanese language version of the Parent Stress Index (PSI, Abidin 1983) was used to assess perceived stress in the mother-child dyad. The PSI yields Child and Parent Domain scores and a Total Stress score. The Japanese version of the measure has good psychometric properties (Narama et al. 1999). Both the Child Domain and Parent Domain scores were used in the current study. Cronbach's alpha for these composite scores was 0.80 and 0.88 respectively.

Parenting style The Parenting Scale (Arnold et al. 1993) is a 30-item self-report questionnaire designed to assess parenting style/discipline practices. The Japanese version of this measure has a two-factor solution and shows good internal consistency for Over-reactivity $0.82 / 0.86$ and low to moderate consistency for Laxness $0.67 / 0.68$ (control/ clinical samples, Itani 2010). Cronbach's alpha in the current sample were 0.84 and 0.78 respectively.
Parenting competence/efficacy The Parenting Sense of Competence Scale (PSOC; Gibaud-Wallston and Wandersman 1978 cited in Johnston and Mash 1989) is a 17-item self-report measure designed to assess parenting self-esteem. Gilmore and Cuskelly (2009) demonstrated the PSOC comprises three interpretable factors: satisfaction in the parental role (Satisfaction), parenting efficacy (Efficacy), and interest in parenting (Interest). In the current sample, Cronbach's alpha for Efficacy and Satisfaction is quite low at 0.55 and 0.50 . The Interest subscale was not used as it includes only three items. Internal consistency for this measure was further explored using an aggregate of both Satisfaction and Efficacy as well as all three factors. This did not improve internal consistency. However, based on the fact consistency was acceptable at $\mathrm{T} 2$ and in a Japanese community sample recruited alongside this study, the measure was included in analysis. The Parental Locus of Control Scale (PLOC, Campis et al. 1986) is a 47-item scale designed to assess parents' perceived locus of control in child-rearing situations. Factor analysis has identified five subscales with adequate internal consistencies (Campis et al. 1986). In this study Cronbach's alpha was 0.43 for Parental Efficacy, 0.69 for Parent Responsibility, 0.55 Child Control of Parent's Life, and 0.69 for Parental Control of Child's Life indicating some factors had low internal consistency that could not be addressed using item deletions. As internal consistency was not better at T2, the Parental Efficacy and Child Control of Parent's life subscales were excluded from the analysis, although the means and standard deviations are still presented in Table 2. The Parent's Belief in Fate/Chance factor was not included in this study as Japanese mothers found the items making up the scale difficult to interpret (Shimabukuro et al. 2017). Both the PSOC and the PLOC were translated into Japanese by the first author and independently back-translated by two bilingual US trained counselling psychologists. There is currently no published Japanese psychometric data for the PSOC or PLOC.

Mood The 21-item 2nd edition of the Beck Depression Inventory (BDI-II, Beck et al. 1996) was used to assess mothers' levels of depression. Items correspond to the diagnostic criteria for depressive disorders in the DSM-IV (APA 2013). Kojima et al. (2002) translated the BDI-II into Japanese and examined its psychometric properties, concluding it is psychometrically robust and can be used to measure depressive symptoms in Japanese populations. In the current sample Cronbach's alpha was 0.91 .

\section{Parent-child interactions}

The quality of mother-child interactions was evaluated through direct observation of behaviour during a cooperative pasta making task. Each mother-child pair 
Table 2 Maternal wellbeing, self-reported parenting, parent-child interactions, and parent report of child behavior: descriptive data, analyses, and significance

\begin{tabular}{|c|c|c|c|c|c|c|c|}
\hline \multirow[t]{2}{*}{ Variable $^{\mathrm{a}}$} & \multicolumn{2}{|c|}{ Immediate Treatment Group } & \multicolumn{2}{|c|}{ Waitlist Control Group } & \multirow[t]{2}{*}{ Covaried F (df) } & \multirow[t]{2}{*}{ Significance } & \multirow[t]{2}{*}{ Cohen's d } \\
\hline & $\begin{array}{l}\text { Time } 1 \\
\text { mean }(\mathrm{SD})\end{array}$ & $\begin{array}{l}\text { Time } 2 \\
\text { mean }(\mathrm{SD})\end{array}$ & $\begin{array}{l}\text { Time } 1 \\
\text { mean }(\mathrm{SD})\end{array}$ & $\begin{array}{l}\text { Time } 2 \\
\text { mean }(\mathrm{SD})\end{array}$ & & & \\
\hline \multicolumn{8}{|l|}{$\mathrm{PSI}^{\mathrm{c}}$} \\
\hline Child domain & $106.19(15.22)$ & $95.22(17.05)$ & $104.20(10.02)$ & $105.45(18.24)$ & $6.911(1.44)$ & 0.012 & 0.776 \\
\hline Parent domain & $118.89(21.10)$ & $102.11(21.42)$ & $119.10(17.51)$ & $122.05(20.97)$ & $16.846(1.44)$ & $<0.001$ & 1.211 \\
\hline BDI & $15.74(10.17)$ & $10.37(8.70)$ & $14.05(7.79)$ & $12.43(9.86)$ & $2.933(1.45)$ & 0.094 & 0.499 \\
\hline \multicolumn{8}{|l|}{ PLOC $^{\mathrm{d}}$} \\
\hline Parent responsibility & $30.44(6.14)$ & $29.33(5.16)$ & $30.48(7.16)$ & $29.76(4.57)$ & $0.096(1.45)$ & 0.758 & 0.090 \\
\hline Parent efficacy & $25.41(4.54)$ & $22.30(3.93)$ & $24.67(4.77)$ & $24.24(3.99)$ & & & 0.504 \\
\hline Child control & $20.96(4.27)$ & $19.15(3.55)$ & $21.38(4.28)$ & $21.00(4.12)$ & & & 0.499 \\
\hline Parent control & $31.52(6.63)$ & $26.37(5.98)$ & $30.60(4.88)$ & $32.15(5.83)$ & $16.997(1.44)$ & $<0.001$ & 1.218 \\
\hline \multicolumn{8}{|l|}{ PSOC $^{\mathrm{e}}$} \\
\hline Satisfaction & $19.19(5.57)$ & $21.78(5.39)$ & $19.29(4.08)$ & $19.38(4.84)$ & $3.831(1.45)$ & 0.057 & 0.569 \\
\hline Efficacy & $12.44(3.84)$ & $15.33(4.86)$ & $14.05(3.81)$ & $13.24(3.82)$ & $5.929(1.45)$ & 0.019 & 0.716 \\
\hline \multicolumn{8}{|l|}{ Parenting Scale } \\
\hline Overreactivity & $44.70(10.05)$ & $30.26(11.23)$ & $42.71(10.34)$ & $42.57(8.51)$ & $34.266(1.45)$ & $<0.001$ & 1.707 \\
\hline Laxness & $22.19(8.16)$ & $19.26(5.67)$ & $24.33(7.66)$ & $24.14(6.85)$ & $8.574(1.45)$ & 0.005 & 0.856 \\
\hline \multicolumn{8}{|l|}{ Parent-child ${ }^{\mathrm{f}}$ interaction } \\
\hline Positive parenting & $8.38(2.82)$ & $9.00(3.37)$ & $8.33(2.28)$ & $7.83(3.26)$ & $1.285(1.41)$ & 0.262 & 0.338 \\
\hline Negative parenting & $5.38(2.61)$ & $4.62(1.13)$ & $4.94(1.16)$ & $5.94(2.51)$ & $4.469(1.41)$ & 0.006 & 0.914 \\
\hline Negative child affect & $2.73(0.92)$ & $2.65(1.16)$ & $2.72(0.96)$ & $2.83(1.14)$ & $0.837(1.41)$ & 0.366 & 0.279 \\
\hline \multicolumn{8}{|l|}{ SNAP } \\
\hline Inattention & 18.89 (3.83) & $14.63(4.91)$ & $19.33(3.95)$ & $17.48(4.53)$ & $4.576(1.45)$ & 0.038 & 0.511 \\
\hline Hyperactivity & $14.15(5.68)$ & $8.89(4.48)$ & $11.62(6.20)$ & $8.52(5.29)$ & $0.789(1.45)$ & 0.379 & 0.261 \\
\hline ODD & $9.70(6.12)$ & $4.67(3.14)$ & $9.67(4.78)$ & $6.14(4.92)$ & $2.240(1.45)$ & 0.141 & 0.435 \\
\hline \multicolumn{8}{|l|}{$\mathrm{CBCL}^{\mathrm{g}, \mathrm{h}}$} \\
\hline Inattention & $65.48(7.11)$ & $62.85(6.91)$ & $65.70(6.15)$ & $66.00(5.62)$ & $4.902(1.44)$ & 0.032 & 0.586 \\
\hline Aggression & $64.26(5.32)$ & $61.26(5.40)$ & $65.15(4.34)$ & $64.20(4.48)$ & $4.391(1.44)$ & 0.042 & 0.619 \\
\hline Internalizing & $59.11(9.85)$ & $55.67(9.19)$ & $62.20(8.60)$ & $62.20(7.00)$ & $6.788(1.44)$ & 0.012 & 0.774 \\
\hline
\end{tabular}

${ }^{a}$ Means and standard deviations based on raw data

${ }^{\mathrm{b}}$ Cohen's d calculated with T2 covaried group means and the pooled standard deviations

cPSI: immediate treatment $n=27$, waitlist $n=20$

${ }^{\mathrm{d}}$ Higher Parent Responsibility, Parent Efficacy, Parent Control = greater sense of competence

${ }^{\mathrm{e}}$ Higher score $=$ greater sense of competence

${ }^{\mathrm{f}}$ Parent-child interactions: immediate treatment $n=26$, waitlist $n=18$

${ }^{\mathrm{g}}$ Means and standard deviations based on $\mathrm{T}$ scores

${ }^{\mathrm{h}} \mathrm{CBCL}$ : immediate treatment $n=26$, waitlist $n=20$

worked together for $15 \mathrm{~min}$ to make pasta using the ingredients and equipment supplied. The interaction was video recorded for later coding. To maintain interest, parent-child pairs made a different type of pasta at each session, counterbalanced across assessments. Interactions were coded using selected parent (rejection and invalidation, coerciveness, negative affect, positive affect, emotional support, parent scaffolding) and child (negative affect, withdrawal, and positive affect) codes from the System for Coding Interactions and Family Functioning (SCIFF, Lindahl and Malik
2000) and the System for Coding Interactions in ParentChild Dyads (SCIPD, Lindahl and Malik 1996). The SCIFF was designed for use with families of different ethnicities and has been used with families of children with ADHD (Lindahl and Malik 2000). To the best of our knowledge, the SCIFF and SCIPD have not previously been used in Japan. Trained raters, blind to the study hypotheses, group membership and task order, coded the interactions. One rater coded all available interactions, a second $20 \%$ selected at random. 
Composite scales positive parenting (positive affect, emotional support, parent scaffolding), negative parenting (negative affect, rejection/invalidation, coerciveness) and negative child affect (withdrawal, child negative affect) were analysed in the current study. Reliability (interclass correlation coefficients) for individual scales ranged from 0.78 (child withdrawal) to 0.90 (parent positive and negative affect) and for the composite scales from 0.84 (negative child affect) to 0.97 (negative parenting).

\section{Data Analysis}

Treatment effects and their precision were quantified with ANCOVA modelling with $\mathrm{T} 1$ scores entered as covariates. Missingness was imputed by means of multiple imputation with a chained equation procedure under a missing at random assumption. Twenty imputed datasets were generated for each outcome. The same analytical model was performed for each outcome using the observed data which included imputed missing data to check the robustness of treatment effects and ensure they were not sensitive to missingness. Missing data represent $2.1 \%$ for those included in the analyses and $9.6 \%$ for those randomised. Missingness did not influence study outcomes. Final statistical analyses were performed with the data from mothers who completed the T1 and T2 assessment measures (treatment $n=27$, control $n=21$ ). Where data violated the assumptions of analysis of variance and/ or there were extreme outliers, data were subject to square root (BDI) or inverse (Negative Parenting, Negative Child Affect) transformations. Sequential multiple regression analyses were performed to confirm the ANCOVA results when the assumption of homogeneity of regression was violated (SNAP ODD scale; PSI Child Domain score; Pasta Task Negative Child Affect scale). Stata 15 and SPSS 21 were used for data analyses. The Hochberg method, a step-up modification of the Bonferroni method (Blakesley et al. 2009), was used to control for familywise error rates within assessment measures. Given the modest sample size, findings significant at $p<0.05$ are acknowledged in the text.

\section{Results}

Table 1 presents the parent and child demographic characteristics for the immediate treatment and waitlist control groups. There were no significant between group differences for any assessed parent (age, education, family structure) or child (age, estimated IQ, gender, medication use, education, ADHD and ODD symptom severity) characteristic.

\section{Maternal Self-Report}

As the study primary outcome variable, the parenting stress results are presented first, followed by the other maternal self-report variables. After completing the Well Parent Japan programme mothers in the treatment group reported significantly less Parent Domain $(F(1,44)=$ 16.85, $p \leq 0.001)$ and Child Domain $(F(1,44)=6.91, p=$ $0.012)$ stress than waitlist control group mothers. They also reported greater perceived control of their child's behaviour (PLOC Parent Control of Child Behavior scale $F(1,44)=16.98, p<0.001)$ and higher levels of parenting efficacy (PSOC Efficacy scale $F(1,45)=5.93, p=0.019$ ), with a trend towards increased parenting satisfaction (PSOC Satisfaction scale $F(1,45)=3.83, p=0.057$ ). Treatment group mothers also reported using more effective parenting strategies compared to those in the waitlist control group, i.e., significantly lower levels of Over-reactivity $(F(1,45)=34.27, p<0.001)$ and Laxness $(F(1,45)=8.57, p<0.001)$ on the Parenting Scale. No other comparisons were significant. Descriptive data and effect sizes for the parent self-report measures are presented in Table 2.

\section{Mother-Child Interactions}

Table 2 also presents the descriptive data and effect sizes for the coded parent-child interactions. Following participation in Well Parent Japan, treatment group mothers were observed to engage in significantly less negative parenting $(F(1,41)=8.47, p=0.006)$ than mothers in the waitlist control group. No other group comparisons were significant however improvements in positive parenting and reductions in negative child affect favoured intervention and yielded effect sizes of around 0.3 .

\section{Maternal Report of Child Behaviour}

Analysis of Covariance of the SNAP and CBCL data indicated participation in Well Parent Japan resulted in lower maternal ratings of inattention on the SNAP $(F(1,45)=4.58, p=0.038)$ and the CBCL Inattention Problems scale $(F(1,44)=4.90, p=0.032)$. Treatment group mothers also reported their children were less aggressive (CBCL Aggression Scale, $F(1,44)=4.39, p=$ 0.042 ) and had fewer internalizing problems (CBCL Internalizing Behavior, $F(1,44)=6.79, p=0.012$ ) than mothers in the wait-list control group. Following correction for multiple comparisons only the aggression and internalizing problems results remained significant. See Table 2 for descriptive data and effect sizes for mothers' reports of child behaviour. 


\section{Discussion}

We used an RCT to evaluate the effectiveness of Well Parent Japan with Japanese mothers of children diagnosed with, or at risk for, ADHD. Well Parent Japan combines a parenting programme of established efficacy in the management of ADHD with strategies known to improve the psychological wellbeing of mothers of children with ADHD (Treacy et al. 2005). As predicted, completing the programme led to significant reductions in parenting stress and maternal reports of children's aggression and internalizing problems and a nonsignificant decrease in inattention. Participation also led to improvements in reported and observed parenting practices and reported parenting self-esteem.

As with previous RCTs of NFPP (Abikoff et al. 2015; Lange et al. 2018; Sonuga-Barke et al. 2001; Thompson et al. 2009), mothers who completed Well Parent Japan reported reduced symptoms of ADHD, in this case inattention, in their children compared with wait-list control group parents. However, these effects did not survive correction for multiple comparisons, despite a moderate effect size. Ratings of Aggression were significantly lower in the treatment group, consistent with reports that the skills taught in parenting programmes decrease children's externalizing behaviour problems (Daley et al. 2018). Together these results offer tentative support for the effectiveness of the parenting skills taught in NFPP, including their generalizability to other cultural contexts, modes of delivery, and developmental stage, i.e., in this study school-aged children.

Mothers' uptake of the skills taught is supported by significant changes in their reported parenting practices. Treatment group participants rated themselves as significantly less over-reactive and lax in dealing with their children's behaviour following intervention. Observations of the parent-child interactions showed negative parenting decreased significantly amongst treatment group mothers, confirming their self-reports of improved parenting and there were trends in favour of intervention for positive parenting also. This objective demonstration of behaviour change is noteworthy as the absence of a follow-up point may have limited the ability to capture the impact of intervention on observed parenting practices and the study had modest statistical power. In addition, previous trials of NFPP report reduced negative expressed emotion, but not observed behaviour change (Thompson et al. 2009).

Consistent with the findings from our earlier proof of concept study, mothers' ratings of child internalizing difficulties were significantly lower following intervention. As with ratings of aggression, this may reflect a decrease in levels of child emotional distress or a change in mothers' perceptions of child behaviour, possibly a result of their decreased parenting stress. Alternatively, a reduction in the children's internalizing problems might follow changes in parenting behaviour, including increased consistency and reduced reactivity/negativity.

Well Parent Japan directly targets parent's emotional wellbeing in addition to teaching parenting skills. Based on the results of our earlier proof of concept study (Shimabukuro et al. 2017), and the work of Treacy et al. (2005), we hypothesized completion of the programme would be accompanied by reductions in parenting stress, and improvements in parenting self-esteem. Mothers completing the programme reported significantly less Parent Domain and Child Domain stress than those in the wait-list group. They also reported increased parenting efficacy and a greater sense of control over their child's behaviour.

The design of the current study does not allow separation of the effects of the additional Well Parent sessions from those of Japanese culturally adapted NFPP. The reductions in Parent Domain stress might reasonably be attributed to these extra sessions. Anecdotally, participating mothers reported the content of these sessions led them to reflect on, and modify their thoughts and behaviours. Treacy et al. (2005) reported similarly large effects on maternal Parent Domain stress in their study, whereas only the initial RCT with NFPP (Sonuga-Barke et al. 2001) described a positive impact on mothers' mental health. Improvements in self-reported parenting style were also described by Treacy et al. (2005) suggesting the Well Parent sessions also contributed to positive change in parenting practices.

Group based programme delivery may also have played a part in improving maternal wellbeing. Feedback from the proof of concept study, and observations from the current RCT group leaders, indicated participants valued hearing other group members' successes and challenges in implementing the skills taught. The group format was selected to provide a support network for mothers while learning and practicing new self-care and parenting skills. This was seen as important to the programme's acceptability in Japan, where difficulties in parenting children with ADHD are not widely voiced. It is also consistent with Japanese collectivism in which a sense of shared experience enhances engagement with, and commitment to, an activity. Furthermore, group-based programmes have proved successful in treating depression and parenting stress in mothers of children with ADHD (Chronis et al. 2006; Chronis-Tuscano et al. 2013; Treacy et al. 2005).

Significant treatment effects were not observed for all outcome variables. On several measures change was in the predicted direction with effect sizes in the moderate range: depression, PSOC parenting satisfaction, and child inattention and ODD symptom severity. The absence of significant group differences on these variables possibly reflecting limited power given the moderate sample size. Sample size does not explain the lack of significant change for the remaining variables. Contrary to the findings of the Abikoff et al. (2015) 
NFPP trial, participation in the current intervention was not accompanied by a significant reduction in child hyperactivity/ impulsivity. Earlier NFPP trials did not separate ADHD symptom types preventing comparisons. In the Abikoff et al. study, levels of inattention and hyperactivity/impulsivity were similar and within the clinical range. In the current sample, mean severity ratings for hyperactivity/impulsivity were between 1 and 2, i.e., occurring more than "Just a Little" but less than "Pretty Much" at baseline, leaving limited room for improvement. These lower scores may reflect the older age of the target children, or the subject pool itself, participating mothers were volunteers rather than referred for parent management training.

The NFPP aims to increase rates of appropriate behaviour through clear communication and the use of positive reinforcement. Despite this, observed levels of positive parenting did not increase significantly over the 13 weeks of the Well Parent Japan programme. As we have previously described, praise is used sparingly in Japanese culture with both mothers and therapists describing this as a challenging aspect of the programme (Shimabukuro et al. 2017).

Despite the increased emphasis on psychoeducation and cognitive restructuring, Japanese mothers completing Well Parent Japan showed little change in their sense of responsibility for their child's behaviour. This may reflect Japanese mothers' roles as primary caregivers responsible for their child's success, and the value placed on persistence of effort. However, Treacy et al. (2005) reported a similar failure to reduce maternal sense of responsibility in New Zealand mothers of children with ADHD. Parents' sense of responsibility for child behaviour may not be readily amenable to change. A more appropriate goal may be helping mothers cope with their feelings of responsibility.

Overall the findings of the current RCT are positive and favour the use of Well Parent Japan as a psychosocial intervention for Japanese mothers parenting children with ADHD. The programme's success in reducing parenting stress and the objective changes in negative parenting support the addition of the Well Parent sessions to the NFPP content. The stronger effects on maternal wellbeing in this trial of NFPP suggest these sessions contributed directly or indirectly to improved maternal emotional functioning.

\section{Limitations and Future Research}

Alongside these encouraging findings it is important to acknowledge the study's limitations. The final sample size is moderate and relatively homogeneous. The latter raising questions about the generalizability of the effects to more impaired or diverse populations. The study was undertaken in a university setting, allowing strict protocol control, including the same well-trained therapists conducting all group sessions. The effectiveness of the programme in less tightly controlled situations remains to be evaluated. The availability of well normed outcome measures in Japanese is limited, making it difficult to assess the clinical relevance of some of the changes identified. As in many parenting studies, outcome data was primarily collected from the participants themselves. The exception in this study being the blind coding of parentchild interactions.

The current study included a waitlist control group but no treatment as usual (TAU) condition. As psychosocial interventions are not routinely available for families of children with ADHD in Japan, we believe the waitlist control group functions as a proxy for TAU in this location. We did not attempt to compare Well Parent Japan with other parenting programmes available in Japan as, to the best of our knowledge, these programs have not been subject to RCTs with families of children with ADHD. The current trial assessed immediate treatment effects only and the study design did not allow an exploration of the independent contribution of parental well-being and NFPP sessions to overall changes in both parent and child behaviour. The low internal consistency of some components of parenting competence is a concern. Similarly, it was necessary to withdraw subscales from the parent locus of control measure due to low internal consistency.

Poor internal consistency for these measures may indicate cultural differences in how these concepts operate within Asian parent samples. For example, Japanese mothers often receive much higher levels of blame for their children's behaviour than western mothers. It would be interesting for future studies to explore the impact of this on both parenting self-esteem and parenting locus of control. Future studies need to evaluate the stability of the observed treatment effects over a longer time period. This would also allow an evaluation of the impact of symptom reduction on children's social and academic functioning. The findings of the earlier proof of concept study would predict maintenance of treatment effects.

Treatment integrity was clearly followed in this trial in that the content delivered was checked at the end of every session and any missing content was added to the beginning of the next session. However, treatment integrity was not independently evaluated after the end of the study. We could strongly encourage other authors to ensure that they plan their trials to allow for an independent evaluation of treatment integrity.

Despite its many limitations, the current trial is the first RCT of a parenting programme targeting ADHD in Japan. Participating in the programme was associated with significant reductions in parenting stress, greater parenting confidence and improved parenting practices, confirmed by direct observation. Participating mothers reported reductions in child aggression, internalizing difficulties and inattention although the latter effect did not survive control for multiple comparisons, although the effect size difference 
was promising in this small sample. Next steps should include a larger effectiveness study that recruits participants from more diverse settings, includes additional blinded outcome measures, assesses the persistence of change over time and explores the additive effects of the parental wellbeing and NFPP sessions.

Acknowledgements The authors thank the participating mothers and children. They also thank Emi Nakanishi and Shoko Nagatomo for assisting with the laboratory assessments, Ryoko Uchida and Kai Ueno for coding the parent child interactions and Dr Boliang Guo for conducting the missingness analysis. The authors acknowledge the NFPP developers, who permitted the modification of NFPP for use with Japanese families.

Author Contributions S.S. screened and recruited mothers for the trial, delivered the intervention, helped with data analysis and co-authored the paper. D.D. advised on the design of the study, outcome measures, helped with data analysis and co-authored the paper. M.T. helped with supervision of the therapist who delivered the intervention, advised on outcomes and co-authored the paper. C.L.-B. helped with supervision of the therapists who delivered the intervention, advised on outcomes and co-authored the paper. K.L. helped to deliver the intervention, assisted with assessment of baseline data and outcome measures and co-authored the paper. G.T. was the principal investigator, held the OIST funding, advised on the design of the study, took a lead on data analysis and co-authored the paper.

Funding This work was supported by a KAKENHI (Grants-in-Aid for Scientific Research) from the Japan Society for the Promotion of Science to S.S. and internal subsidy funding from the Okinawa Institute of Science and Technology Graduate University (OIST), Okinawa, Japan.

\section{Compliance with Ethical Standards}

Conflict of Interest K.L. and G.T. have no interests to declare. S.S. has received a speaker fee from Shire. D.D. received fees from Eli Lilly, non-financial support from Eli Lilly, grants from Shire, personal fees from Shire, non-financial support from Shire, fees from Medice, nonfinancial support from HBPharma and royalties from the sale of the new Forest Parent Training Programme Self Help book. M.T. has received recent funding from Shire, a speaker fee from Jansen-Cilag, fees from training and supervision of the New Forest Parenting Programme and royalties from the sale of the new Forest Parent Training Programme Self Help book. C.L.-B. has received speaker fees from Janssen-Cilag and Shire, fees from training and supervision of the New Forest Parenting Programme and royalties from the sale of the New Forest Parent Training Programme Self Help book.

Ethical Approval This study was approved by the Okinawa Institute of Science and Technology (OIST) Graduate University, Japan Human Subjects Research Review Committee.

Informed Consent Participating mothers provided written consent to participate and children written assent.

Publisher's note Springer Nature remains neutral with regard to jurisdictional claims in published maps and institutional affiliations.
Open Access This article is licensed under a Creative Commons Attribution 4.0 International License, which permits use, sharing, adaptation, distribution and reproduction in any medium or format, as long as you give appropriate credit to the original author(s) and the source, provide a link to the Creative Commons license, and indicate if changes were made. The images or other third party material in this article are included in the article's Creative Commons license, unless indicated otherwise in a credit line to the material. If material is not included in the article's Creative Commons license and your intended use is not permitted by statutory regulation or exceeds the permitted use, you will need to obtain permission directly from the copyright holder. To view a copy of this license, visit http://creativecommons. org/licenses/by/4.0/.

\section{Appendix 1: Well Parent Japan Session Content}

\section{Session Content}

\begin{tabular}{|c|c|c|}
\hline 1 & $\begin{array}{l}\text { Orientation to program. } \\
\text { What is ADHD? Part } 1\end{array}$ & Psychoeducation \\
\hline 2 & $\begin{array}{l}\text { Stress management, } \\
\text { relaxation }\end{array}$ & $\begin{array}{l}\text { Mothers } \\
\text { emotional health }\end{array}$ \\
\hline 3 & Cognitive restructuring & \\
\hline & Problem solving & \\
\hline & Communication skills & \\
\hline & What is ADHD? Part 2 & Psychoeducation \\
\hline
\end{tabular}
Recruiting attention, positive communication, praise (earshotting)

$7 \quad$ Zone of proximal development, choices, clear messages, countdowns, using a timer

$8 \quad$ Review of session 7 skills, use of play

9 House rules, routine, boundaries, reward and punishment

10 Review of session 9, review of ADHD symptoms

11 Temper tantrums (time out, quiet time), anticipating, avoiding conflict

12 Emotion and language, child relaxation

13 Social stories, mindfulness, wrap-up session 


\section{References}

Abidin, R. (1983). Parenting stress index-manual. Charlottesville, VA: Pediatric Psychology Press.

Abikoff, H. B., Thompson, M., Laver-Bradbury, C., Long, N., Forehand, R. L., Brotman, L. M., Klein, R. G., Reiss, P., Huo, L., \& Sonuga-Barke, E. (2015). Parent training for preschool ADHD: a randomized controlled trial of specialized and generic programs. Journal of Child Psychology \& Psychiatry, 56, 618-631. https:// doi.org/10.1111/jcpp.12346.

Achenbach, T. (1991). Manual for the child behavior checklist/4-18 and 1991 profile. Burlington, VT: University of Vermont.

American Psychiatric Association (2013). Diagnostic and statistical manual of mental disorders. (5th ed.). Arlington, VA: American Psychiatric Publishing.

Arnold, D. S., O’Leary, S. G., Wolff, L. S., \& Acker, M. M. (1993). The parenting scale: a measure of dysfunctional parenting in discipline situations. Psychological Assessment, 5, 137-144. https://doi.org/10.1037/1040-3590.5.2.137.

Beck, A. T., Steer, R. A., \& Brown, G. K. (1996). Beck Depression Inventory-II. San Antonio, TX: The Psychological Corporation.

Barkley, R. (2013). Taking charge of ADHD: the complete authoritative guide for parents. (3rd ed.). Boystown, NE: The Guilford Press.

Barlow, J., Smailagic, N., Huband, N., Roloff, V., \& Bennett, C. (2014). Group-based parent training programmes for improving parental psychosocial health. Cochrane Database of Systematic Reviews, (5), CD002020. https://doi.org/10.1002/14651858.CD002020.pub4.

Blakesley, R. E., Mazumdar, S., Dew, M. A., Houck, P. R., Tang, G., Reynolds, III, C. F., \& Butters, M. A. (2009). Comparisons of methods for multiple hypothesis testing in neuropsychological research. Neuropsychology, 23(2), 255-264. https://doi.org/10. 1037/a0012850.

Campis, L. K., Lyman, R. D., \& Prentice-Dunn, S. (1986). The parental locus of control scale: development and validation. Journal of Clinical Child Psychology, 15(3), 260-267. https://doi.org/10. 1207/s15374424jccp1503_10.

Chronis, A. M., Gamble, S. A., Roberts, J. E., \& Pelham, W. E. (2006). Cognitive-behavioral depression treatment for mothers of children with attention-deficit/hyperactivity disorder. Behavior Therapy, 37 (2), 143-158. https://doi.org/10.1016/j.beth.2005.08.001.

Chronis-Tuscano, A., Clarke, T. L., O’Brien, K. A., Raggi, V. L., Diaz, Y., Mintz, A. D., Rooney, M. E., Knight, L. A., Seymour, K. E., Thomas, S. R., Seeley, J., Kosty, K., \& Lewinsohn, P. (2013). Development and preliminary evaluation of an integrated treatment targeting parenting and depressive symptoms in mothers of children with attention-deficit/hyperactivity disorder. Journal of Consulting and Clinical Psychology, 81(5), 981-925. https://doi.org/10.1037/a0032112.

Daley, D., \& Birchwood, J. (2010). ADHD and academic performance: why does ADHD impact on academic performance and what can be done to support ADHD children in the classroom? Child Care Health \& Development, 36(4), 455-464. https://doi. org/10.1111/j.1365-2214.2009.01046.x.

Daley, D., Van Der Oord, S., Ferrin, M., Cortese, S., Danckaerts, M., Doepfner, M., Van den Hoofdakker, B. J., Coghill, D., Thompson, M., Asherson, P., Banaschewski, T., Brandeis, D., Buitelaar, J., Dittmann, R. W., Hollis, C., Holtmann, M., Konofal, E., Lecendreux, M., Rothenberger, A., Santosh, P., \& Sonuga-Barke, E. J. (2018). Practitioner review: current best practice in the use of parent training and other behavioural interventions in the treatment of children and adolescents with attention deficit hyperactivity disorder. Journal of Child Psychology \& Psychiatry, 59(9), 932-947. https://doi.org/10.1111/jcpp.12825.
Daley, D., Van Der Oord, S., Ferrin, M., Danckaerts, M., Doepfner, M., Cortese, S., \& Sonuga-Barke, E. J. S. (2014). Behavioral interventions in attention-deficit/hyperactivity disorder: a metaanalysis of randomized controlled trials across multiple outcome domains. Journal of the American Academy of Child \& Adolescent Psychiatry, 53(8), 835-847. https://doi.org/10.1016/j.jaac. 2014.05.013.

Gilliam, J. E. (2013). GARS-3: Gilliam Autism Rating Scale (3rd ed.). Austin, TX: Pro-ed.

Gilmore, L., \& Cuskelly, M. (2009). Factor structure of the parenting sense of competence scale using a normative sample. Child Care Health \& Development, 35(1), 48-55. https://doi.org/10.1111/j. 1365-2214.2008.00867.x.

Inoue, Y., Ito, K., Kita, Y., Inagaki, M., Kaga, M., \& Swanson, J. M. (2014). Psychometric properties of Japanese version of the Swanson, Nolan, and Pelham, version-IV scale-teacher form: a study of school children in community samples. Brain Development, 36(8), 700-706. https://doi.org/10.1016/j.braindev.2013. 09.003 .

Itani, T. (2010). The Japanese version of the parenting scale: factor structure and psychometric properties. The Japanese Journal of Psychology, 81(5), 446-452. https://doi.org/10.4992/jjpsy.81. 446.

Itani, T., Kanbayashi, Y., \& Nakata, Y. (2001). Development of child behavior checklist/4-18 Japanese version. Psychiatrica et Neurologia Japonica, 41(4), 243-252.

Iwasaka, H. (2012). Parenting children with difficulties by using praise: parent training guidebook. Jiho Inc. (In Japanese, title translated by the first author of this article).

Japanese Ministry of Education, Culture, Sports, Science and Technology. (2012). The research report on children who attend a regular class but possibly have a developmental disorder and need a special need education. https://www.mext.go.jp/a_menu/shotou/ tokubetu/material/icsFiles/afieldfile/2012/12/10/1328729_01.pdf. (In Japanese, title translated by the first author of this article).

Johnston, C., \& Mash, E. J. (1989). A measure of parenting satisfaction and efficacy. Journal of Clinical Child Psychology, 18(2), 167-175. https://doi.org/10.1207/s15374424jccp1802_8.

Kojima, M., Furukawa, T. A., Takahashi, H., Kawai, M., Nagaya, T., \& Tokudome, S. (2002). Cross-cultural validation of the Beck Depression Inventory-II in Japan. Psychiatry Research, 110(3), 291-299. https://doi.org/10.1016/S0165-1781(02)00106-3.

Lange, A. M., Daley, D., Frydenberg, M., Houmann, T., Kristensen, L. J., Rask, C., Sonuga-Barke, E., Sondergaard-Baden, S., Udupi, A., \& Thomsen, P. H. (2018). Parenting training for preschool ADHD in routine, specialist care: a randomized controlled trial. Journal of the American Academy of Child and Adolescent Psychiatry, 57(8), 593-602. https://doi.org/10.1016/j.jaac.2018. 04.014.

Laver-Bradbury, C., \& Harris, H. (2009). Advanced nurse practitioners for attention deficit hyperactivity disorder (ADHD). London, UK: Department of Health.

Lindahl, K. M., \& Malik, N. M. (1996). System for coding interactions in parent-child dyads (SCIPD). Miami, FL: University of Miami.

Lindahl, K. M., \& Malik, N. (2000). The system for coding interactions and family functioning. In P. K. Kerig \& K. M. Lindahl (Eds). Family observational coding systems: resources for systemic research (pp. 77-92). Philadelphia, PA: Brunner/Mazel.

Matsumoto, Y., Sofronoff, K., \& Sanders, M. R. (2007). The efficacy and acceptability of the Triple P-Positive parenting program with Japanese parents. Behaviour Change, 24(4), 205-218. https://doi. org/10.1375/bech.24.4.205.

Matsumoto, Y., Sofronoff, K., \& Sanders, M. R. (2010). Investigation of the effectiveness and social validity of the Triple $\mathrm{P}$ positive 
parenting program in Japanese society. Journal of Family Psychology, 24(1), 87-91. https://doi.org/10.1037/a0018181.

Mikami, A. Y., Huang-Pollock, C., Pfiffner, L. J., McBurnett, K., \& Hangai, D. (2007). Social skills differences among attention-deficit/ hyperactivity disorder types in a chat room assessment task. Journal of Abnormal Child Psychology, 35(4), 509-521. https://doi.org/10. 1007/s10802-007-9108-5.

Narama, M., Kanematsu, Y., Araki, A., Maru, M., Nakamura, N., Takeda, J., Shirahata, N., \& Kudo, Y. (1999). Validity and reliability of the Japanese version of the parenting stress index. The Journal of Child Health, 58(5), 610-616. (In Japanese, title translated by the first author of this article).

National Institute for Health and Care Excellence. (2018). Attention deficit hyperactivity disorder: diagnosis and management NICE guideline [NG87]. National Institute for Health and Care Excellence. https://www.nice.org.uk/guidance/ng87.

Okuma, H., \& Ito, K. (2005). Hizen Style Parenting Training Program learning room for parents of children with $A D / H D$. Niheisha. (In Japanese, title translated by the first author of this article).

Sakakibara, Y. (2007). Brain science and developmental disordersthe mechanism known up to now. Tokyo: Chuohouki Publishing. (In Japanese, title translated by the first author of this article).

Sanders, M. R., Markie-Dadds, C., \& Turner, K. M. T. (2003). Theoretical, scientific and clinical foundations of the Triple P-positive parenting program: a population approach to the promotion of parenting competence. Parenting Research and Practice Monograph, 1, 1-24. https://www.expoo.be/sites/default/files/atoms/ files/artikelTripleP.pdf.

Shaw, M., Hodgkins, P., Caci, H., Young, S., Kahle, J., Woods, A. G., \& Arnold, L. E. (2012). A systematic review and analysis of long-term outcomes in attention deficit hyperactivity disorder: effects of treatment and non-treatment. BMC Medicine, 10, 99. https://doi.org/10.1186/1741-7015-10-99.

Shimabukuro, S., Daley, D., Thompson, M., Laver-Bradbury, C., Nakanishi, E., \& Tripp, G. (2017). Supporting Japanese mothers of children with ADHD: cultural adaptation of the New Forest Parent Training Programme. Japanese Psychological Research, 59(1), 35-48. https://doi.org/10.1111/jpr.12140.

Sonuga-Barke, E. J. S., Barton, J., Daley, D., Hutchings, J., Maishman, T., Raftery, J., Stanton, L., Laver-Bradbury, C., Chorozoglou, M., Coghill, D., Little, L., Ruddock, M., Radford, M., Yao, G., Lee, L., Gould, L., Shipway, L., Markomichali, P., McGuirk, J., Lowe, M., \& Thompson, M. J. J. (2018). A comparison of the clinical effectiveness and cost of specialised individually delivered parent training for preschool attention-deficit/hyperactivity disorder and a generic, group-based programme: a multi-centre, randomised controlled trial of the New Forest Parenting Programme versus
Incredible Years. European Child \& Adolescent Psychiatry, 27, 797-809. https://doi.org/10.1007/s00787-017-1054-3.

Sonuga-Barke, E. J. S., Brandeis, D., Cortese, S., Daley, D., Ferrin, M., Holtmann, M., Stevenson, J., Danckaerts, M., Van Der Oord, S., Dopfner, M., Dittmann, R. W., Simonoff, E., Zuddas, A., Banaschewski, T., Buitelaar, J., Coghill, D., Hollis, C., Konofal, E., Lecendreux, M., \& Wong, I. C. K., the uropean ADHD Guidelines Group. (2013). Non-pharmacological interventions for attention-deficit hyperactivity disorder: systematic review and meta-analyses of randomized controlled trials of dietary and psychological treatments. The American Journal of Psychiatry, 170(3), 275-289. https://doi.org/10.1176/appi.ajp. 2012.12070991.

Sonuga-Barke, E. J. S., Daley, D., Thompson, M., Laver-Bradbury, C., \& Weeks, A. (2001). Parent-based therapies for preschool attention-deficit/hyperactivity disorder: a randomized, controlled trial with a community sample. Journal of the American Academy of Child \& Adolescent Psychiatry, 40(4), 402-408. https://doi. org/10.1097/00004583-200104000-00008.

Sonuga-Barke, E. J. S., Thompson, M., Abikoff, H., Klein, R., \& Brotman, L. M. (2006). Nonpharmacological interventions for preschoolers with ADHD - the case for specialized parent training. Infants \& Young Children, 19(2), 142-153. https://doi.org/ 10.1097/00001163-200604000-00007.

Swanson, J. M. (1992). School-based assessments and interventions for ADD students. Irvine, CA: KC Publishing.

Takayama, K. (2008). Problems of Western style AD/HD parenting training program: trial of parent support program adapted to Japanese culture. Japanese Journal of Pediatrics, 61, 2504-2510. (In Japanese, title translated by the first author of this article).

Theule, J., Wiener, J., Tannock, R., \& Jenkins, J. M. (2013). Parenting stress in families of children with ADHD: a meta-analysis. Journal of Emotional and Behavioral Disorders, 21(1), 3-17.

Treacy, L., Tripp, G., \& Baird, A. (2005). Parent stress management training for attention-deficit/hyperactivity disorder. Behavior Therapy, 36(3), 223-233. https://doi.org/10.1037/a0032112.

Thompson, M. J. J., Laver-Bradbury, C., Ayres, M., Poidevin, E. L., Mead, S., Dodds, C., Psychogiou, L., Bitsakou, P., Daley, D., Weeks, A., Brotman, L. M., Abikoff, H., Thompson, P., \& Sonuga-Barke, E. J. S. (2009). A small-scale randomized controlled trial of the revised New Forest Parenting Programme for preschoolers with attention deficit hyperactivity disorder. European Child \& Adolescent Psychiatry, 18(10), 605-616. https:// doi.org/10.1007/s00787-009-0020-0.

Wechsler, D. (2010). Japanese version of the Wechsler Intelligence Scale for children (4th ed.). Tokyo: Nihon Bunka Kagaku.

Whitham, C. (1998). Win the whining war and other skirmishes: a family peace plan. London: Perspective Publishing. 